\title{
STUDIES IN TEMPERATURE SENSATION. I. A COMPARISON OF THE SENSATION PRODUCED BY INFRA-RED AND VISIBLE RADIATION
}

\author{
BY THEODORE W. OPPEL 1 AND JAMES D. HARDY \\ (From the Russell Sage Institute of Pathology in affiliation with the New York Hospital, and \\ the Department of Medicine, Cornell University Medical College, New York City)
}

(Received for publication January 6, 1937)

Few of the studies of temperature sensation have emphasized the importance of the perception of thermal change on the regulation of internal body temperature. Not only does the recognition of such changes make it possible for a man to provide a more comfortable environment for himself, but the automatic regulation of heat production and heat loss is dependent primarily upon it. The separate channels through which the human body dissipates its heat have been extensively studied and each of them has been shown to 'be important under certain environmental conditions. The heat exchange between man and objects in his environment can be altered by increasing or decreasing the rate of blood flow to the skin. The resulting alteration of skin temperature varies the heat exchange by conduction, convection, vaporization, and radiation; and so balances the external heat loss against the heat produced by metabolism that a constant internal temperature is maintained. The recognition by the organism of the temperature of environmental objects is thus important for the regulation of body temperature.

The temperature receptors in the skin which are responsible for the sensation of warmth and cold have been identified (1), and although these end organs are doubtless concerned in the autonomic control of skin temperature, there is no proof that other end organs may not be equally important. However, the study of temperature sensation is the most convenient way of investigating the perception of environmental temperature change.

The knowledge of temperature sensation as it is ordinarily experienced is meagre because almost all experiments have been performed by placing hot or cold objects on the skin so that only the effect of conducted heat or cold has been determined. Such thermal stimulation is always combined with tactile sensation and is quite different

\footnotetext{
1 New York Hospital Research Fellow.
}

from the stimulation produced by a change of environmental convection or radiation. These latter changes are experienced much more frequently than those due to contact with warm or cold bodies.

There are many reasons why radiant heat is the most suitable stimulus for a study of temperature perception. It is the only thermal stimulus which can be applied without simultaneously provoking another sensation. The magnitude of the stimulus is readily controlled and measured, and the accompanying skin temperature elevation can be determined. The duration of the radiation and the size of the area to which it is applied are unlimited. The use of penetrating radiation has made it possible to produce different types of thermal gradient change during heating and has proved useful in establishing the mechanism by which the end organs in the skin are stimulated by heat. Using radiant heat we have investigated several aspects of temperature perception and have discussed them in the following three parts : Part I considers the absorption of radiation by the skin and the relative effectiveness of radiations of different wavelength on the stimulation of warm sensation. Part II deals with the thermal changes in the skin which are responsible for the sensation of warmth. Part III discusses the minimum sensitivity of the body to temperature change and the effect of the size of the irradiated area on the sensation produced, as it is influenced by the number of end organs stimulated and by spatial summation of the separate end organ responses.

Of all forms of thermal stimuli, radiation is the most frequent and physiologically the most important. Changes in environmental radiation are constantly taking place and a radiation stimulus always precedes a conduction stimulus whenever there is a marked difference of temperature. Furthermore, recent studies (4) have shown that 
the exchange of radiation between the body surface and objects in the environment is the most important mechanism of heat loss under ordinary conditions. Under conditions of comfort about 60 per cent of the heat produced by the body is lost by radiation, and this amount varies considerably under extremes of environmental radiation. In zero weather a man loses twice as 'much heat by radiation as he produces under basal conditions; and on exposure to the sun on a clear, hot day he absorbs more than three times as many calories as his basal heat production (12). The response to changes of environmental radiation is in itself, therefore, one of the most important mechanisms of heat regulation.

The radiation to which man is exposed in his natural environment comprises a spectral range from $0.29 \mu$ in the ultraviolet to $20 \mu$ in the infrared. Physiologically, this range may be divided into four regions, the ultraviolet $(\lambda<.36 \mu)$, the visible $(0.36 \mu<\lambda<0.8 \mu)$, the penetrating infra-red $(0.8 \mu<\lambda<3 \mu)$, and the non-penetrating infra-red $(\lambda>3 \mu)$. The infra-red spectrum is so divided because of the intense absorption at the skin surface of radiation longer than $3 \mu$. This absorption is due to the presence of water and organic compounds in the skin (8). The action of ultraviolet light upon the skin is largely photochemical, and this type of radiation does not occur naturally in sufficient amounts to affect the thermal mechanism of the body. The effect of the longer waved radiation, in so far as is known, is entirely thermal. The sun, which is the most important radiator in man's environment, has an energy distribution at the 'earth's surface on a clear day about as follows $(11,13): 5$ per cent ultraviolet, 40 per cent visible light, 54 per cent penetrating 'infra-red, and 1 per cent nonpenetrating infra-red. All other bodies in man's surroundings radiate penetrating infra-red or nonpenetrating infra-red.

The degree of heating of the skin by radiation depends on the quantity of energy absorbed and therefore is influenced by the reflecting power and penetrability of the skin. These physical properties of the skin have been studied for various spectral regions. Hardy and Muschenheim (9) found that white human skin reflected less than 5 per cent of the non-penetrating infra-red radia- tion, and that 90 per cent of the energy was absorbed within $0.05 \mathrm{~mm}$. of the skin surface. The reflection of penetrating infra-red was greater, as much as 25 per cent of the short wave near infrared being reflected and nearly 50 per cent of the remaining energy penetrating to a depth of 0.5 $\mathrm{mm}$. For both regions, the properties of the negro skin for reflection and penetration were found to be the same as those for the white skin. From white skin, more visible than penetrating infrared radiation was reflected, and visible radiation has been shown to penetrate deeper than penetrating infra-red (2). Negro skin reflects about half as much visible radiation as white skin; the penetration of negro skin has not been satisfactorily measured, but it is probably more opaque than white skin to visible radiation.

A sensory effect of far infra-red, near infrared, and visible radiation was studied by Sonne (14). He exposed an area on the forearm of white subjects to the maximum amount of radiation from these sources which the subject could bear, and measured the intensity in $\mathrm{gm}$. $\mathrm{cal} / \mathrm{cm}^{2} / \mathrm{min}$. The values were for visible 3.11 , for penetrating infra-red 1.79 , and for non-penetrating red 1.33. He found that the skin reflected 35 per cent of the visible and penetrating infra-red radiation and none of the longer waved infra-red radiation.

Keller (10) determined the effect of artificial pigment on the heating resulting from irradiation by these sources. He found that painting the skin with india ink had no effect on the heating due to non-penetrating infra-red radiation. However, the same quantity of visible radiation produced a much greater rise in the surface temperature after the pigment was applied than before. The effect of india ink on the heating due to penetrating infra-red radiation was similar to, but less marked than that due to visible radiation.

The present experiments differ from those of Sonne and Keller in several ways. The minimum perceptible sensation has been studied because we believe that it is a more delicate test of warmth perception than the maximum bearable sensation which is essentially painful. Instead of applying pigment to white subjects, negro subjects have been used as a more direct approach to the prob- 
lem of pigment function. In addition to the artificial sources of radiation sunlight has been used.

\section{METHODS}

The sources of artificial radiation which we employed were chosen after careful spectrometric analysis and their emission curves are shown in Figure 1. Non-penetrating infra-red radiation of longer wavelength than $3 \mu$ was obtained from a 250 watt electric hot plate, $12 \mathrm{~cm}$. in diameter. A thousand watt tungsten filament flood light furnished a penetrating infra-red band between $0.8 \mu$ and $3 \mu$ after the visible and long wave infra-red had been filtered out by a Corning heat transmitting glass. This lamp also supplied the visible radiation from $0.4 \mu$ to $0.7 \mu$ after the light'had passed through $9 \mathrm{~cm}$. of an 0.8 per cent aqueous solution of copper sulphate. This filter did not completely absorb a small band of infra-red at $1 \mu$ but it furnished the most complete source of pure visible light we were able to obtain. The near infra-red constituted head of the subject $\mathrm{H}$ was held behind a small screen in which a circular aperture, $14.5 \mathrm{~cm}^{2}$, limited an area on the forehead to irradiation. A large screen separated the subject from the operator and allowed the settings for the stimuli to be made without the subject's knowledge. A cardboard shutter was held between the source and a hole in this screen so that rays could pass to the subject only when this shutter was removed. During experiments with'visible radiation, special care was taken that the subject did not see the light.

A test for the minimum intensity of radiation which would produce sensation was made in the following way. The subject sat behind the screen with his head in position for some minutes before these experiments began, to accustom himself to the thermal sensation of his forehead. The shutter was then removed and a stimulating amount of radiation allowed to fall on the exposed skin surface for 3 seconds. This length of time was arbitrarily chosen because it was found that if any sensation were produced it would be evoked by irradiation for this

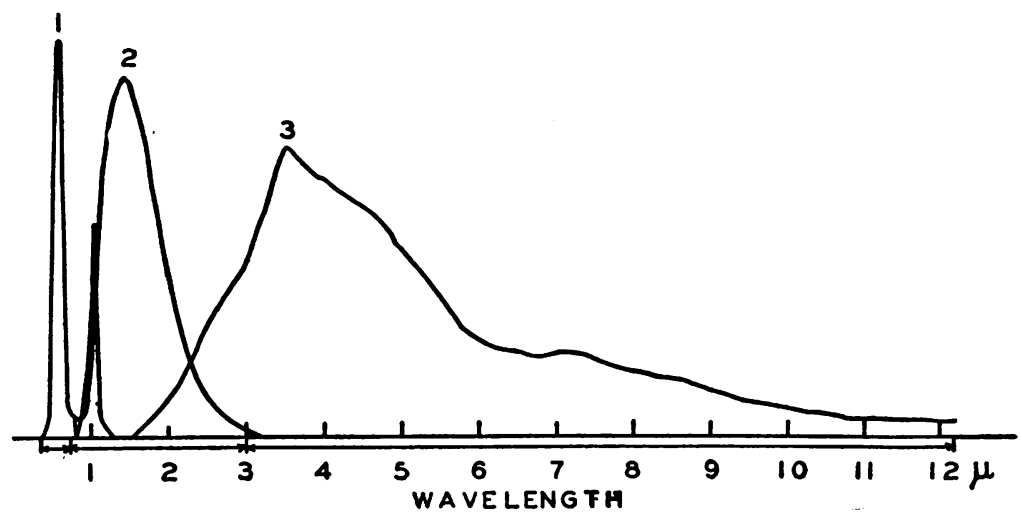

Fig. 1. Spectral Distributions of the Energies of the Artificial Sources of Radiation: (1) Visible Light, (2) Penetrating Infra-Red, (3) Non-Penetrating INFra-Red

about 24 per cent of the transmitted energy, and attempts to decrease the relative amount of near infra-red by altering the thickness or concentration of the filter resulted in absorption of the visible red rays. To obtain a sufficient intensity of visible, and in some cases of penetrating infra-red, the radiation had to be concentrated by a lens. Precautions were taken to keep the focused radiation as uniform as possible over the exposed skin surface. The intensity of various parts of the field was measured, and the maximum variation of intensity was 10 per cent.

The sunlight used for stimulation was directed into the laboratory by a heliostat. The radiation was reflected from two second surface silvered mirrors, and its intensity was regulated by controlling the size of the effective aperture.

A diagram of the apparatus used to measure the stimulating effect of radiation is shown in Figure 2. The source of the radiation, $S$, was mounted on a roll table so that the strength of the stimulus could be altered by moving the source toward or away from the subject. The time. The subject signalled his perception by calling " on." The intensity of radiation was then decreased by steps, and the test repeated until the smallest intensity of radiation was found to which the subject responded with accuracy. The forehead was then removed, and the radiometer, $R$, placed in the aperture in the screen to measure the strength of the radiation. This strength was designated the minimum stimulus.

False sensations of warmth were largely eliminated by noting the time after the onset of irradiation at which the subject called " on." With radiation of the minimum perceptible strength, the sensation was always noted about 3 seconds after the exposure began. By this means the operator evaluated the perception of sensation reported by the subject. The test was considered satisfactory when the minimum radiation was found to which the subject responded in the characteristic time interval each time he was exposed to radiation. Fatigue and lack of concentration caused a great decrease in the sensitivity of subjects, but when these factors were controlled similar 
values were obtained on repeated tests. This method is more objective than those previously used in the study of warm sensations.

The radiometer used to measure the intensity of radiation was fitted with a small metal flange which rested against the surface of the screen in such a way that the where

$R \mathrm{MgCO}_{3}=$ Percentage reflected by $\mathrm{MgCO}_{3}$,

$R$ skin $=$ Percentage reflected by skin,

$d \quad=$ Galvanometer deflection caused by radiation reflected by $\mathrm{MgCO}_{3}$,

$d_{1} \quad=$ Galvanometer deflection caused by radiation reflected by the skin.

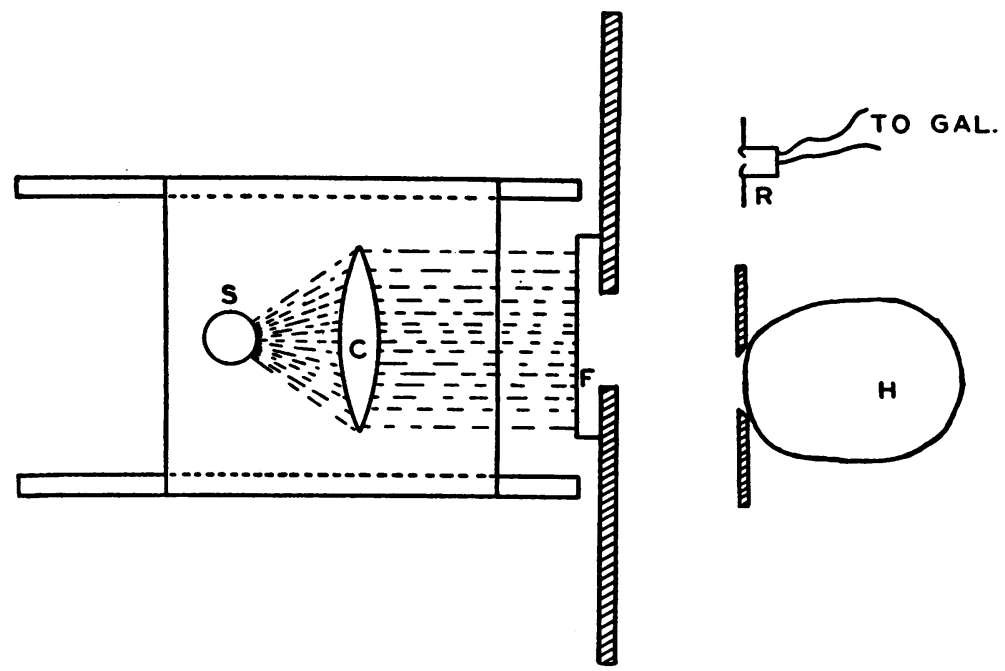

Fig. 2. Diagram of Apparatus for Measuring the Minimum DeteCtable ENERGY

$S=1000$ watt lamp; $C=$ condensing lens $F=$ filter $H=$ subject's head; $R=$ radiometer.

absorbing surface of the instrument was always located in the plane of the skin, perpendicular to the beam of radiation. The thermopile of this instrument which was constructed in our laboratory was of the type previously described (6) except that the radiation receivers were blackened with india ink. The voltage generated was measured with a Leeds and Northrup potentiometer (Type $\mathrm{K}-2$ ). The calibration was carried out with a Leslie Cube and checked against a radiation standard of the National Bureau of Standards. The sensitivity of the instrument was such that $0.000051 \mathrm{gm}$. $\mathrm{cal} / \mathrm{cm}^{2} / \mathrm{sec}$. of radiation generated 0.00001 volt.

To measure the reflecting power of the skin the forehead was placed in the aperture in the screen and a very sensitive radiometer adjusted $7 \mathrm{~cm}$. from the skin, perpendicular to its surface. The galvanometer index was set at zero to compensate for the natural radiation from the skin. The skin was then irradiated from an angle of about $60^{\circ}$ from the normal with a strength of radiation too weak to change the skin temperature. The galvanometer deflection resulting from radiation diffusely reflected into the radiometer was measured. The procedure was repeated, substituting a scraped block of magnesium carbonate for the skin surface. Reflection from the skin was calculated from the proportion:

$$
\frac{R \mathrm{MgCO}_{3}}{R \text { skin }}=\frac{d}{d_{1}},
$$

The reflection of various spectral regions for magnesium carbonate has been measured by Coblentz (3). He found that about 86 per cent of radiation was reflected throughout the visible spectrum. We determined penetrating infra-red reflection by interpolating Coblentz's values for our source and obtained the result 73 per cent. The percentage of non-penetrating infra-red reflection from skin was too small to measure by the above method. Since it is known that both white and negro skin reflects less than 5 per cent of far infra-red beyond $2 \mu$ we have made no correction for non-penetrating infra-red reflection.

\section{RESULTS}

The smallest intensity of radiation, measured in gram calories per second per $\mathrm{cm}^{2}$, capable of producing a sensation was determined on twelve white subjects. They showed moderate differences of sensitivity but the relative stimulating amounts of visible, penetrating infra-red, and non-penetrating infra-red radiation were the same for all. The results of tests on three white subjects are characteristic of the group and are shown in Table I. Subjects I and II were males (with fair skins); Subject III was a woman (whose skin had the same reflecting power as that 
TABLE I

Minimum stimulus in $\mathrm{gm}$. cal $/ \mathrm{cm}^{2} / \mathrm{sec} .:$ White subjects

\begin{tabular}{|c|c|c|c|c|c|c|c|c|c|}
\hline \multirow[b]{2}{*}{ Subject............ } & \multicolumn{3}{|c|}{ Visible light } & \multicolumn{3}{|c|}{$\begin{array}{l}\text { Penetrating } \\
\text { infra-red }\end{array}$} & \multicolumn{3}{|c|}{$\begin{array}{c}\text { Non-penetrating } \\
\text { infra-red }\end{array}$} \\
\hline & I & II & III & I & II & III & I & II & III \\
\hline $\begin{array}{l}\text { Average for } 3 \text { sub- } \\
\text { jects.............. } \\
\text { Reflecting power of } \\
\text { skin, per cent..... } \\
\text { Energy absorbed... }\end{array}$ & $\begin{array}{l}.0026 \\
.0025 \\
.0025 \\
.0028 \\
.0026\end{array}$ & $\begin{array}{c}.0026 \\
.0027 \\
\\
.0027 \\
35 \\
.0018\end{array}$ & \begin{tabular}{|l|}
.0028 \\
.0029
\end{tabular} & $\begin{array}{l}.0015 \\
.0014 \\
.0015\end{array} \mid$ & $\begin{array}{c}.0014 \\
.0014 \\
.0015 \\
.0015 \\
\\
.0014 \\
20 \\
.0011\end{array}$ & .0012 & $\begin{array}{l}.0008 \\
.0008 \\
.0009\end{array}$ & $\begin{array}{c}.0011 \\
.0009 \\
.0009 \\
.0008 \\
0 \\
.0008\end{array}$ & $\begin{array}{l}.0005 \\
.0005\end{array}$ \\
\hline
\end{tabular}

of the other two subjects). An area of $14.5 \mathrm{sq}$. $\mathrm{cm}$. of forehead was exposed to the radiation.

It will be seen that the sensitivity of white subjects increases with the wavelength of the incident radiation so that the smallest response is produced by the visible light and the greatest by the nonpenetrating infra-red. The reflecting power of white skin for these radiations is also given in the table and even when allowance is made for the energy reflected, the increase in sensitivity with increasing wavelength is apparent. Thus, the absorption by the skin of the same amount of visible, penetrating, or non-penetrating infra-red will not produce the same intensity of sensation.

The sensitivity of two negroes to the three types of radiation was measured. The values are re-

TABLE II

Minimum stimulus in $\mathrm{gm} . \mathrm{cal} / \mathrm{cm}^{2} / \mathrm{sec} .:$ Negro subjects

\begin{tabular}{|c|c|c|c|c|c|c|}
\hline \multirow[b]{2}{*}{ Subject............. } & \multicolumn{2}{|c|}{ Visible light } & \multicolumn{2}{|c|}{$\begin{array}{l}\text { Penetrating } \\
\text { infra-red }\end{array}$} & \multicolumn{2}{|c|}{$\begin{array}{c}\text { Non- } \\
\text { penetrating } \\
\text { infra-red }\end{array}$} \\
\hline & I & II & I & II & I & II \\
\hline 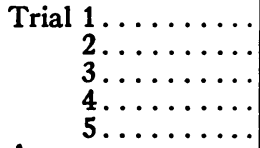 & $\begin{array}{l}.0012 \\
.0017 \\
.0011 \\
.0013\end{array}$ & $\begin{array}{l}.0015 \\
.0014 \\
.0012\end{array}$ & $\begin{array}{l}.0016 \\
.0019 \\
.0019 \\
.0020 \\
.0020\end{array}$ & $\begin{array}{l}.0020 \\
.0020\end{array}$ & $\begin{array}{l}.0009 \\
.0013 \\
.0012 \\
.0012\end{array}$ & $\begin{array}{l}.0006 \\
.0006\end{array}$ \\
\hline $\begin{array}{l}\text { Average ......... } \\
\text { Skin reflecting }\end{array}$ & .0013 & .0014 & .0019 & .0020 & .0011 & .0006 \\
\hline $\begin{array}{l}\text { power, per cent. } \\
\text { Energy absorbed. }\end{array}$ & $\begin{array}{r}13 \\
.00\end{array}$ & $11^{16}$ & $\begin{array}{l}17 \\
.001\end{array}$ & $15^{19}$ & 0 & 99 \\
\hline
\end{tabular}

corded in Table II. Subject I was a very dark negro man; Subject II was a woman of a medium brown color. The difference in pigmentation is evidenced by the skin reflecting power. As the pigmentation of negroes is highly variable the values given in Table II would not apply to all colored subjects.
TABLE III

Minimum stimuli in $\mathrm{gm}$. cal/ $\mathrm{cm}^{2} / \mathrm{sec}$. of sun's radiation

\begin{tabular}{|c|c|c|c|c|c|}
\hline \multirow[b]{2}{*}{ Subject................. } & \multicolumn{3}{|c|}{ White subjects } & \multicolumn{2}{|c|}{ Negro subjects } \\
\hline & $\mathbf{I}$ & II & III & $\mathbf{I}$ & II \\
\hline 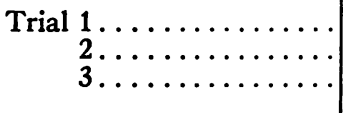 & $\begin{array}{l}.0020 \\
.0026 \\
.0026\end{array}$ & $\begin{array}{l}.0023 \\
.0026 \\
.0026\end{array}$ & $\begin{array}{l}.0020 \\
.0025 \\
.0028\end{array}$ & $\begin{array}{l}.0025 \\
.0021 \\
.0019 \\
.0027\end{array}$ & $\begin{array}{l}.0023 \\
.0024 \\
.0029\end{array}$ \\
\hline $\begin{array}{l}\text { Average................ } \\
\text { Reflecting power, per cent } \\
\text { Energy absorbed........ }\end{array}$ & .0025 & $\begin{array}{c}.0025 \\
30 \\
.0018\end{array}$ & .0024 & $\begin{array}{c}.0023 \\
17 \\
.00\end{array}$ & $\begin{array}{l}.0025 \\
19 \\
20\end{array}$ \\
\hline
\end{tabular}

The thermal response of the white and negro subjects to sunlight was compared. It was found convenient to use a larger area of the forehead for these measurements and a skin area of $23.8 \mathrm{~cm}^{2}$ was irradiated. The minimum stimulus was measured in the usual way, and the values are given in Table III. The sensitivity of white and negro subjects to the incident sunlight is seen to be approximately the same; the negro subjects required 'greater absorption of energy to produce sensation.

\section{COMMENT}

The results (Table I) of the study of the sensitivity of white subjects to radiations of different wavelength show that part of this effect is due to the difference in the reflecting power of the skin for the various radiations. After allowing for reflected energy the non-penetrating infra-red is more than twice as effective as the visible light in producing sensation, and it is evident that the effectiveness of the radiation in stimulating sensation depends upon the opacity of the skin for that radiation. The smaller the penetrating power of the rays the more easily they produce sensation. That the stimulating effect of radiation depends solely on reflection and penetration can be seen from the following experiment. The skin of white subjects was coated with india ink to provide a surface which completely absorbs all radiation, and minimum stimuli were determined by exposing this artificially pigmented skin. The sensitivity of the subjects to all the radiation bands was then the same and was equal to that of the non-penetrating, non-reflecting infra-red radiation. Thus, all the differences in the response of white skin to these radiations can be attributed to a combination of reflection and penetration of the energy. 
The ratio of the amounts of these radiations which Sonne found when he produced the maximum bearable sensation in white subjects, is practically the same as that for the barely perceptible warmth. It thus appears likely that this ratio is valid for all degrees of sensation produced by radiation. The sensation which Sonne (14) produced was essentially that of pain and not of heat, a fact which suggests that strong stimulation of the heat receptors results in pain. negro's pigment influences the absorption of radiation and the sensitivity to radiation. Studies with carbon black are not applicable to an understanding of the functions of the human skin pigment as related to radiation $(5,10)$.

The relationship between the absorption of radiation by the skin and the sensitivity of both white and negro subjects to it is shown diagrammatically on Figure 3 . The basis for the comparison is the sensitivity of white subjects to

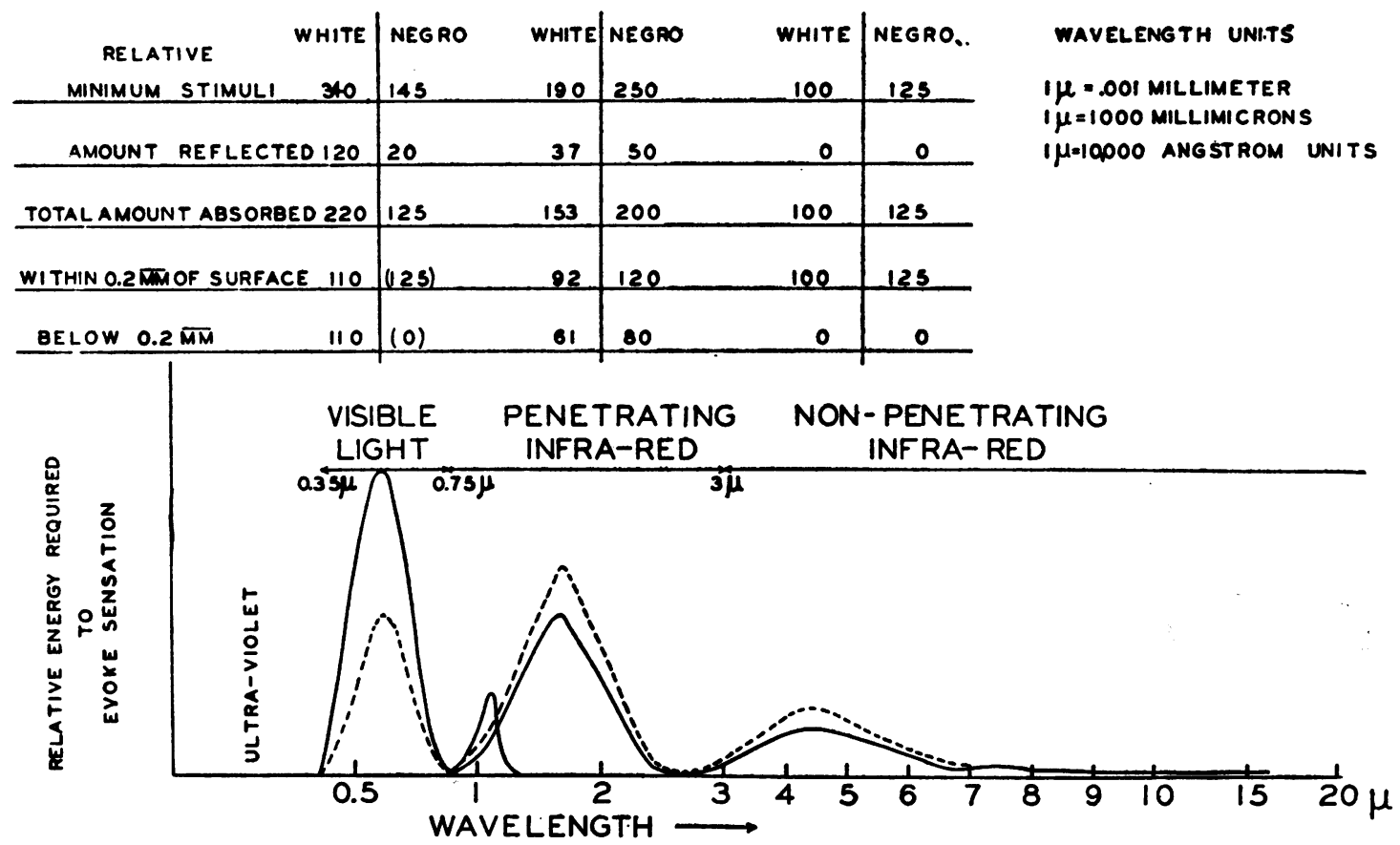

Fig. 3. Comparative Study of Response of White and Negro Subjects to Various Radiation Bands. Solid Line: White Subjects. Interrupted Line: Negro Subjects

Minimum stimuli for negroes (Table II), as for white subjects, depend on the reflecting and penetrating properties of the skin. Negro skin is more opaque than white skin to visible radiation and to it the negroes were 55 per cent more sensitive than the white subjects. For both types of infra-red radiation white and negro skin have the same absorbing properties, and the minimum stimuli for these two radiation bands show the same ratio on the two groups of subjects. The fact that the negroes required about 25 per cent more of both infra-red radiations than the white subjects is best accounted for by a difference in the individual sensitivity of the subjects to heat. It is only for the visible spectral region that the non-penetrating infra-red; it is assumed that 100 units of this radiation are required for stimulation of sensation. For each type of radiation the minimum stimulating quantity has been divided into the amount reflected and the amount absorbed, and the latter has been subdivided into that absorbed at the skin surface (superficial $0.2 \mathrm{~mm}$.) and that which penetrates into the skin. In the lower part of the figure minimum stimuli have been plotted'as solid curves for the white subjects and as interrupted curves for the negroes. The only marked difference in these two curves is for visible light and this is associated with a striking difference in the corresponding part of the table. That visible radiation does not penetrate into 
negro skin as it does into white skin is shown by this diagram. The absorption by the negroes of only 125 units of visible radiation is required to produce sensation; this is the same as the stimulating amount of non-penetrating infra-red radiation. Since non-penetrating infra-red is completely absorbed within the superficial $0.2 \mathrm{~mm}$. of the skin it is assumed that the visible radiation is similarly absorbed. Because the penetration of visible light into negro skin has not been measured directly, these values on Figure 3 for the energy absorbed at the skin surface and below, are enclosed in parentheses.

In the experiments with sunlight, the white subjects were stimulated by the absorption of about 10 per cent less radiation than the negroes. If allowance is made for the fact that the white subjects were individually about 25 per, cent more sensitive to heat than the negroes, the negroes are shown to be about 15 per cent more sensitive to solar radiation than the whites. The 40 per cent visible radiation in sunlight is responsible for this difference in the sensitivity of the two groups. Thus only in sunlight can pigment be considered to play a significant rôle in the exchange of body heat by radiation. It was previously shown that pigment has no effect on the loss of heat from the skin surface (7).

The fact that the least penetrating radiation is the most stimulating, indicates that the production of sensation by radiation results from the activation of the temperature receptors in the skin. It has been suggested by Bazett and McGlone (1) that the sensory response to radiation is due to the stimulation of end organs other than those which usually respond to thermal change. No real evidence has been offered for this theory, and the results of the present study exclude such a possibility. The non-penetrating infra-red which is the most stimulating type of radiation is completely absorbed at the skin surface (within 0.1 mm.) and none of it penetrates to the depths at which the end organs are found. The sensory response to this radiation is necessarily due to heat conducted from the surface.

\section{SUMMARY AND CONCLUSIONS}

1. Measurement of the thermal sensitivity of the skin to radiation by a new technique is described. The method provides for an objective determination of the smallest rate of irradiation which will produce sensation. The response of the skin to sunlight, visible light, and infra-red radiation was studied. The infra-red spectrum was studied in two portions, that of wavelength shorter than $3 \mu$ designated as penetrating infrared, and that of longer wavelength designated as non-penetrating infra-red. Artificial sources of visible' light, penetrating, and non-penetrating infra-red, were obtained by use of proper filters and light sources. The purity of the spectral bands was determined by a spectrometer. The reflecting power of the skin and the intensity of the stimulating energy were measured by a radiometer.

2. The specific stimulating qualities of the various radiations were measured for white and negro subjects. The results with white subjects showed that the minimum amounts of incident radiation required to evoke sensation were in the ratio of $3: 2: 1$ respectively, for the visible, penetrating infra-red, and non-penetrating infra-red radiation. Allowing for the reflected energy the ratio became 2.2:1.5:1. This last difference is attributed entirely to the penetrating power of the radiations; the more penetrating the rays, the less sensitive is the subject to them.

The study of negro subjects gave 1.5:2.5:1.3 as the ratios of the minimum stimulating energies on the same basis as the white subjects. Correcting for reflection $1.3: 2.0: 1.3$ was the ratio. In this study also the difference in the stimulating abilities of the radiation bands can be explained by the difference in the opacity of the skin for the radiation.

3. Comparative studies of white and negro subjects showed that the negro subjects were approximately 76 per cent as sensitive to infra-red radiation as the white subjects. The white subjects were 15 per cent less sensitive than dark negroes to the thermal effects of sunlight.

4. The effect of natural pigment upon the response to thermal radiation is significant only in the visible 'portion of the spectrum. The effect of natural pigment upon the thermal response to sunlight is found to be small. Therefore, pigment plays only a small rôle in the thermal exchange of man and his environment. 
5. The sensory response to radiation is the result of the stimulation of the temperature receptors in the skin.

\section{BIBLIOGRAPHY}

1. Bazett, H. C., and McGlone, B., Studies in sensation. II. The mode of stimulation of cutaneous sensations of cold and warmth. Arch. Neurol. and Psychiat., 1932, 27, 1031.

2. Bachem, H., and Reed, C. I., The penetration of light through human skin. Am. J. Physiol., 1931, 97, 86.

3. Coblentz, W. W., The diffuse reflective power of various substances. Bull. U. S. Bureau of Standards, 1913, 9, 283.

4. Du Bois, E. F., Mechanism of heat loss in health and disease. Tr. A. Am. Physicians, 1936, 51, 252.

5. Foster, P. C., Effect of infrared on tissue gradient as influenced by pigment. Proc. Soc. Exper. Biol. and Med., 1935, 33, 62.

6. Hardy, J. D., The radiation of heat from the human body. I. An instrument for measuring the radiation and surface temperature of the skin. J. Clin. Invest., 1934, 13, 593.

7. Hardy, J. D., The radiation of heat from the human body. III. Human skin as a black-body radiator. J. Clin. Invest., 1934, 13, 615.
8. Hardy, J. D., and Muschenheim, C., Radiation of heat from human body. IV. The emission, reflection and transmission of infra-red radiation by the human skin. J. Clin. Invest., 1934, 13, 817.

9. Hardy, J. D., and Muschenheim, C., Radiation of heat from the human body. V. Transmission of infra-red radiation through skin. J. Clin. Invest., $1936,15,1$.

10. Keller, P., The Strahlen-Wärmereaktion der Haut. III. Pigment- und Wärmestrahlenschutz. Strahlentherapie, 1929, 35, 353.

11. Kimball, H. H., and Hand, I. F., The intensity of solar radiation as received on the surface at the earth and its variations with latitude, altitude, the seasons of the year, and the time of day. Chapter II, Duggar, B. I., Biological Effects of Radiation. McGraw-Hill Book Company, New York, 1936.

12. Martin, C. J., Thermal adjustments of man and animals to external conditions. Lancet, 1930, 2, 673.

13. Laurens, H., The Physiological Effects of Radiant Energy. Chapter 2. Chemical Catalog Company, New York, 1933.

14. Sonne, C., The mode of action of the universal light bath. II. Determination of the amount of heat radiated to the skin of luminous and obscure heat rays that can be borne without causing combustion. Acta med. Scandinav., 1921, 54, 350. 